\title{
Impact of standardization of in-ward urine output measurement in post - operative patients: a clinical audit
}

\author{
M. H. Prashath ${ }^{1}$, G. S. Sandhakumari ${ }^{2}$, R. C. Siriwardana ${ }^{2}$ \\ ${ }^{1}$ Professorial Surgical Unit, NCTH, Ragama. \\ ${ }^{2}$ Department of Surgery, Faculty of Medicine, University of Kelaniya.
}

Keywords: Postoperative care; urine outputa audit

\begin{abstract}
Urine output (UOP) is a key parameter used in post-operative patients to monitor the recovery. In our setup, this is done by patients or caregivers. An audit cycle was carried out to develop and evaluate a standardized protocol to improve the accuracy of UOP measurement. The pre-audit assessment was done evaluating: individual's ability in measuring and record keeping, characteristics of the measuring container and error of measurement. Subsequently, corrections were done in these areas. The cycle was completed comparing the error of measurement as an outcome. The intervention group had fewer errors compared to the pre-audit group. Errors can be overcome by a standardized measuring protocol.
\end{abstract}

\section{Introduction}

Variation of urine output is a valuable surrogate marker of impending acute kidney injury (AKI) and tissue perfusion (1). Accurate measurement of urine output is also important in maintaining proper fluid balance in critically ill patients (2).

;In our setup, most of the patients after major surgery are managed in general wards. In our ward setup, the urine output (UOP) is measured usually by caretakers. No standardized methods are followed in the process of measurement. The usual practice is to visually read the recording in the urinecollecting bag and record it. Such readings are usually inaccurate. Errors can happen during urine measuring container manipulation, visual assessment and manual data recording. Errors that happen during measuring, monitoring and recording UOP can end up in delays of identification and early intervention of episodes of oliguria (3) hence clinical diagnosis and decision making.

We observed these drawbacks in our practice as well. An audit cycle was carried out focusing on standardizing the measurement of urine output of inward post-surgical patients.

Correspondence: Prof. Rohan Chaminda Siriwardana

E-mail: rohansiriwardana@yahoo.com

Received: 13-09-2019 Accepted: 14-10-2019

(iD) https://orcid.org/0000-0002-6477-2072 DOI: http://doi.org/10.4038/sljs.v37i4.8637
Methodology

\section{Study sample}

This clinical audit was carried out for 03 months. Twenty-five post-operative patients were included in the pre-audit assessment and another 25 post-operative patients were included in the re-audit cycle. Based on the first cycle results, intervention protocol was prepared to focus on standardizing the process. Accuracy of the urine output measurements was prospectively audited.

\section{Inclusion and exclusion criteria}

The primary inclusion criterion was patients undergoing major surgeries needing postoperative measurement of urine output. Patients younger than 18 years, the patient who can't read numbers, patients with visual impairments and patients who didn't give the consent to participate were excluded.

\section{First audit cycle}

In 25 ( $n=25)$ post-operative patients (group A) as the first step, using an interviewer-administered questionnaire data was collected on, individuals (patient's or bystander's) knowledge on volume reading from the measuring cup (figure 1), writing numbers, record keeping of volume. An interviewer observed the device used for measurements (whether it is a calibrated measuring container or not). Further interviewer assessed the volume reading ability and knowledge on numbers by giving pre-measured water sample and asking them to measure the volume and record it.

To measure the accuracy of measurement, patient or bystander was asked to measure the UOP for next 24 hours duration. Urine was collected to a separate large container given by the interviewer. After 24 hours, interviewer assessed the true volume of urine. As the outcome, the difference between true volume and patient's measurement was recorded. The difference over $50 \mathrm{ml}$ was considered significant.

\section{Intervention protocol}

Based on the findings three interventions were done. An information sheet was given to the patient/ caregiver as a step by step guide on the measuring process. Separate volume 
recording chart indicating hours was prepared to document the UOP. Measurement of urine output was done by clearly calibrated volume measuring cups.

\section{Re-audit cycle}

Another 25 post-operative patients (group B, $\mathrm{n}=25$ ) participated in the re-audit cycle. Same data were collected in group B as described above.

\section{Results}

In the pre-audit group (group A) among 25 patients $(\mathrm{n}=25)$ there were 2 patients $(8 \%)$ who didn't measure UOP at all. Caregiver measured majority (91.3\%). Some patients' $(17.4 \%)$ UOP was measured by more than a single caregiver as they changed within 24 hours. $65.2 \%$ had inadequate knowledge on volume reading from the measuring cup, while all had writing skills. $87 \%$ patients used non-standardized measuring cups. In group A, 87\% of patients had over $50 \mathrm{ml}$ error.

After standardization of the process of measurement, education of the patient and correction of the device, group B had $10 \%$ of patients with over $50 \mathrm{ml}$ error of measurement $(p<0.001)$. According to the results, there was a significant reduction of error after the intervention.

\section{Discussion}

This audit shows that a simple instructions sheet, using a calibrated monitoring cup and providing a standard recording sheet significantly increases the accuracy of measuring the urine output. In a limited resource setup like in Sri Lanka patients, themselves or caregivers play a major role in the post-operative monitoring, unlike other countries where more accurate measuring devices like automatic sensing devices, electronic urinary output monitors are used (4).

UOP is a sensitive surrogate marker for AKI in postoperative patients. This provides an early warning signal for impending renal dysfunction (5). Inaccuracies in measuring urine output can affect clinical diagnosis and subsequent management of post-operative complications. Precise measurement of UOP will give the clinician a chance of timely intervention when necessary. Comparable issues were reported in other places as well. Hersch et.al evaluated the errors committed by the nursing staff when they take measurements visually compared to mechanical measurement. An average visual measurements error has been reported to be as high as $26 \%$ (6).

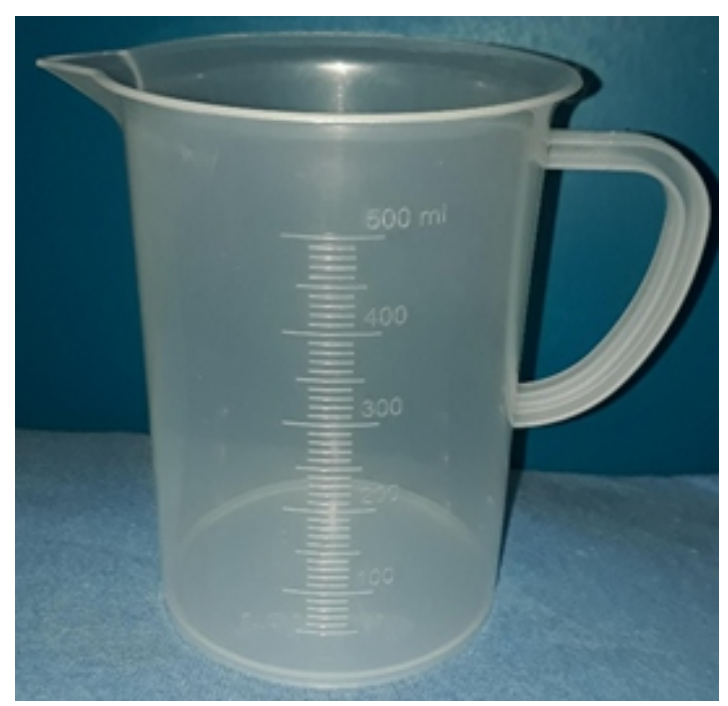

Figure 1. Standardized measuring container

The intervention we made was a simple change that can make a significant difference in accurate monitoring of postoperative patients. Adapting this strategy in our clinical setup in different units needs to be considered.

All authors disclose no conflict of interest. The study was conducted in accordance with the ethical standards of the relevant institutional or national ethics committee and the Helsinki Declaration of 1975, as revised in 2000 .

\section{References}

1. Chenitz KB, Lane-Fall MB. Decreased urine output and acute kidney injury in the PACU. Anesthesiol Clin. 2012 Sep; 30(3): 513-526. https://doi.org/10.1016/j.anclin.2012.07.004

2. Legrand M,Payen D.Understanding urine output in critically ill patients. Ann Intensive Care. 2011 May 24; 1(1): 13. https://doi.org/10.1186/2110-5820-1-13

3. Macedo E, Malhotra R, Granado RC, Fedullo P, Mehta RL. Defining urine output criterion for acute kidney injury in critically ill patients. Nephrol Dial Transplant. 2011 Feb;26(2):509-15. https://doi.org/10.1093/ndt/gfq332

4. Otero A, Palacios F, Akinfiev T, Apalkov A. A low cost device for monitoring the urine output of critical care patients. Sensors (Basel). 2010;10(12):10714-32. https://doi.org/10.3390/s101210714

5. Macedo E, Malhotra R, Bouchard J, Wynn SK, Mehta RL. Oliguria is an early predictor of higher mortality in critically ill patients. Kidney Int. 2011 Oct; 80:760-767. https://doi.org/10.1038/ki.2011.150

6. Hersch M, Einav S, Izbicki G. Accuracy and ease of use of a novel electronic urine output monitoring device compared with standard manual urinometer in the intensive care unit. J Crit Care. 2009 Dec;24(4): 629.e13-7.

https://doi.org/10.1016/j.jcrc.2008.12.008 